\title{
Enfermedad tiroidea y embarazo
}

\author{
Dres. Gustavo Gómez T.*, Rafael A. Camacho**, \\ Lilian González***
}

RESUMEN. En la Clínica de Embarazo de Alto Riesgo Obstétrico-Endocrino se manejaron un total de 52 pacientes con enfermedad tiroidea y embarazo. 30 hipertiroideas, 17 hipotiroideas y 5 con Bocio Eutiroideo. Los diagnósticos principales en hipertiroidismo fueron E. de Graves (66\%) y tiroiditis (16\%). Dos pacientes $(6.6 \%)$ presentaron crisis hipertiroidea y hubo una muerte materna (19\%). Incidencia de $23 \%$ de cesáreas (la mitad por sufrimiento fetal). Dos recién nacidos (14\%) tuvieron diagnóstico de bajo peso al nacer; no hubo muertes perinatales y sí un aborto de 20 semanas. El diagnóstico principal en hipotiroidismo fue tiroiditis de Hashimoto $(30 \%)$. No se presentó mortalidad materna ni fetal. El Bocio Eutiroideo se comporta normalmente durante el embarazo.

Se revisa el tema desde el punto de vista fisiológico durante el embarazo y se discute el diagnóstico y tratamiento.

SUMMARY. 52 patients with tyroid disease and pregnancy are reported. Thirty with hipertyroidism, 17 hypotyroidism and 5 patients with Eutyroid goiter. The two more frequently diagnosis in the hypertyroid group were Graves Disease (66\%) and tyroiditis $(16 \%)$. Two patients $(6.6 \%)$ had hypertyroid crisis and there was one maternal death $(19 \%) .23 \%$ required cesarean section, half of them for fetal distress. Two neonates were small for gestational age. There were not perinatal deaths but one 20 weeks gestation abortion. Hashimoto disease was the principal diagnosis in hypotyroidism group (30\%). There were not maternal niether fetal death in the hypotyroid group.

Finally, we review the physiology and treatment of tyroid disease during pregnancy.

\section{Enfermedad tiroidea y embarazo}

La disfunción de la glándula tiroidea es más común en la mujer, con relación al hombre, en una proporción de 4:1.

Se ha reconocido una íntima relación entre la glándula tiroides y el embarazo. La glándula es alterada por las demandas metabólicas y hormonales del embarazo y a su vez éste puede ser alterado de una manera importante por cambios en la función tiroidea.

\section{Fisiología tiroidea materna}

Durante el embarazo hay un evidente estado hiperdinámico en la madre. Esto puede llevar a confusión con un estado de hiperfunción de la tiroides. Es común encontrar taquicardia, aumento del gasto cardíaco, con un aumento de amplitud del pulso y a veces soplo sistólico, eritema palmar, intolerancia al calor y fatiga.

La glándula tiroidea normal acumula yodo de la dieta a una velocidad constante. Este es incorporado a la hormona tiroidea o sus precursores. El yodo sobrante es excretado

\footnotetext{
* Profesor Asociado. Coordinador Endocrinología Ginecológica e Infertilidad. Departamento de Obstetricia y Ginecología. Universidad del Valle. Cali - Colombia.

** Ex-Residente Departamento de Obstetricia y Ginecología. Universidad del Valle.

*** Ex-Residente Departamento de Obstetricia y Ginecología. Universidad del Cauca. Rotante por el Departamento de Obstetricia y Ginecología de la Universidad del Valle.
}

por los riñones. Posteriormente las hormonas tiroideas son excretadas a la circulación o almacenadas en los acinos tiroideos.

Las hormonas tiroideas, tiroxina (T4) principalmente y también triyodotironina (T3), son transportadas en la circulación por proteínas transportadoras. Estas proteínas son: globulina transportadora de hormonas tiroideas, TBG, prealbúmina transportadora de hormonas tiroideas, TBPA, y albúmina, A (1). La capacidad de enlace de la TBG aumenta significativamente durante el embarazo. De $25 \mathrm{mcg} / \mathrm{dl}$ se incrementa a $50 \mathrm{mcg} / \mathrm{dl}$. Esto explica el aumento de los niveles de hormonas tiroideas medidas por radioinmunoensayo durante el embarazo.

Una vez las hormonas tiroideas llegan a la célula, la fracción libre, no unida a la proteína, puede entrar a la célula. A nivel celular la tiroxina (T4) se deiodiniza a triyodotironina (T3) o en algunas circunstancias a T3 reverso (T3r). La mayoría de T3 proviene de conversión periférica de T4, por deiodinación de ésta en la posición 5'. En el feto hay un bloqueo de la 5 ' deiodinasa y un aumento de la 5 deiodinasa, por lo tanto se produce mayor conversión a T3 reverso. Aunque la actividad calorigénica de T3 y T4 parecen ser similares, la primera se une mucho menos a las proteínas y por lo tanto la fracción libre de la hormona es maycr.

La síntesis y liberación de hormona tiroidea es regulada por mecanismos de retroalimentación negativa que involucran la hipófisis y el hipotálamo. La hormona liberadora de tirotropina TRH, es un tripéptido hipotalámico que estimula 
la liberación de la hormona estimulante del tiroides o TSH (2-3). La TSH a su vez, estimula la captación de yodo, la síntesis de las hormonas tiroideas, también como su liberación, por proteolisis, de la tiroglobulina. Bajo circunstancias normales, los niveles de TSH y el efecto estimulante de TRH, es controlado por mecanismos de retroalimentación negativo de las hormonas tiroideas circulantes.

\section{Hipertiroidismo durante la gestación}

La incidencia de hipertiroidismo durante el embarazo es bajo. Sin embargo, la tirotoxicosis no tratada en la mujer embarazada, está asociada con una alta incidencia de toxemia, muerte fetal y eventualmente, muerte materna (4). El embarazo normal está asociado a menudo con síntomas compatibles con hipertiroidismo, por lo tanto es necesario establecer el diagnóstico a través de exámenes de laboratorio. El hecho que durante el embarazo la TBG está aumentada, da como resultado que tanto T3 como T4 se encuentran normalmente incrementadas. La captación de T3 por una resina (T3 uptake) mide indirectamente los niveles de TBG. La relación de T4 con T3 uptake, configuran el índice de T4 libre, que revela los niveles de la hormona libre. Desde un punto de vista práctico podemos considerar que niveles superiores a $15 \mathrm{mcg} / \mathrm{dl} \mathrm{de} \mathrm{T} 4$ y $220 \mathrm{ng} / \mathrm{dl}$ de T3, indican un aumento real de hormonas tiroideas circulantes (14). Bajo circunstancias especiales, cuando es importante hacet el diagnóstico de hipertiroidismo y los niveles hormonales tiroideos están en el límite, la respuesta de TSH circulante a la administración de TRH, es de utilidad (5).

\section{Hipotiroidismo durante la gestación}

Se ha descrito como una entidad rara durante el embarazo (7). Se encuentra una alta incidencia de infertilidad y anovulación hipogonadotrópica, generalmente relacionada con aumento de la Prolactina. Probablemente hay un incremento de la incidencia de abortos en esta enfermedad (6). Estudios recientes sobre desarrollo del embarazo en pacientes hipotiroideas muestran un pronóstico favorable (6). Lo anterior es explicable porque el feto no necesita de las hormonas tiroideas maternas, puesto que tan temprano como la semana 9-10 de embarazo ya produce sus propias hormonas tiroideas (20-21).

El más sensible indicador de hipotiroidismo son los niveles de TSH, que cuando están elevados hacen el diagnóstico aún en presencia de T4 normal. En este último caso se encuentra lo que denominamos hipotiroidismo subclínico, puesto que la paciente puede estar libre de los síntomas característicos de la enfermedad como son: intolerancia al frío, constipación, adinamia, mixedema, y postración, en orden de gravedad.

El hipotiroidismo a menudo está asociado a tiroiditis de Hashimoto, que puede ser de incidencia familiar. La determinación de anticuerpos, antiglobulinas y antimicrosomales, son útiles para establecer el diagnóstico.

\section{Pacientes}

A la Clínica de Embarazo de Alto Riesgo, EAR, del Hospital Universitario del Valle, HUV, en la sección de Endocrinología del Embarazo, son remitidas y centralizado el manejo de las pacientes embarazadas con trastornos endocrinológicos concomitantes. Los resultados perinatales y el manejo de 54 pacientes con enfermedad tiroidea y embarazo es el objeto de la presente comunicación.

30 pacientes fueron hipertiroideos, 5 presentaban bocio eutiroideo y 17 se diagnosticaron como hipotiroideas (Figura 1). $1)$.

$$
\text { Figura } 1
$$

ENFERMEDAD TIROIDEA Y EMBARAZO

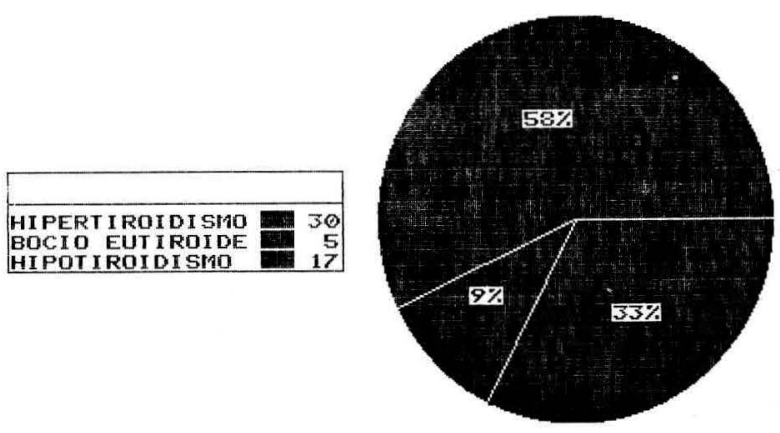

\section{Hipertiroidismo}

De 30 pacientes con diagnóstico de hipertiroidismo, 20 tuvieron diagnóstico clínico y/o patológico de Enfermedad de Graves; 3 fueron catalogadas como Hipertiroidismo; 5 se diagnosticaron Tiroiditis con comprobación patológica; 1 Bocio Nodular parenquimatoso y otra como Nódulo Tiroideo Hiperfuncionante (Figura 2).

Figura 2

HIPERTIROIDISMO Y EMBARAZO

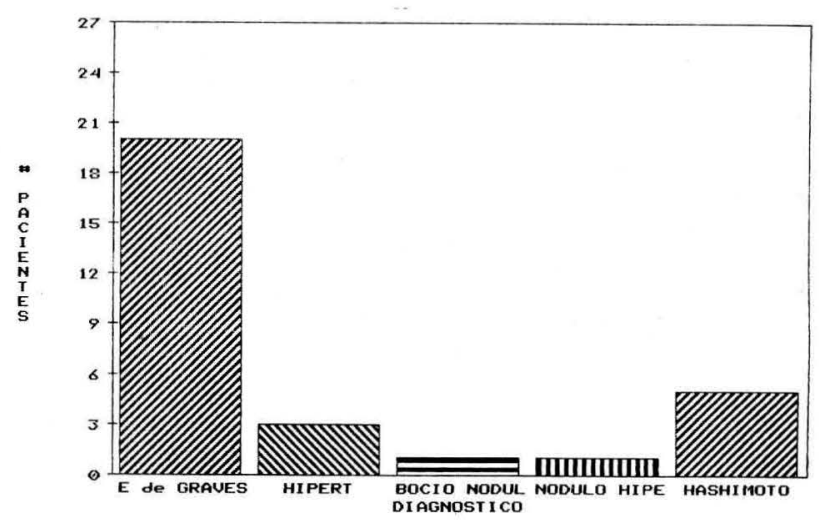

Dos pacientes con Enfermedad de Graves requirieron hospitalización, con diagnóstico de crisis hipertiroidea, después de haber abandonado la medicación antitiroidea. Una de ellas después de 20 días de hospitalización murió de falla cardíaca irreversible. Se trataba de una paciente de 29 años, G1PO con antecedente de hipertensión arterial e hipertiroidismo previo al embarazo. Tenía prescrito $60 \mathrm{mgs}$ de metimazol y $80 \mathrm{mgs}$ de propanolol, con lo que estaba bien 
controlada. Dejó la medicación y a su ingreso presentaba pulso de 130, exoftalmos, ritmo de galope, ingurgitación yugular, $39^{\circ} \mathrm{C}$ de temperatura, anemia, $180 / 100$ de presión arterial. En el examen de ingreso se le encontró bocio difuso, palidez de piel y mucosa, útero grávido para 20 semanas, feto vivo, longitudinal y cefálico, FCF 150 por minuto; en miembro inferior izquierdo presentaba una úlcera hipertensiva o de Martorel infectada, que consideramos, desencadenó la crisis hipertiroidea en la paciente. Los diagnósticos de ingreso fueron: a) hipertiroidismo descompensado; b) ICC de gasto alto; c) HTA crónica; d) edema pulmonar agudo; e) úlcera de Martorel. El tratamiento inicial fue a base de lanicor, lasix, líquidos controlados, antibióticos y reposo absoluto. También se administró lugol 3 gotas cada 6 horas por 24 horas y metimazol hasta $60 \mathrm{mgs}$ diarios. Una vez compensada cardiovascularmente, se agregó propanolol 80-120 mgs en 24 horas. Después de varios días estable, súbitamente entra en descompensación cardíaca que no es posible revertir.

La otra paciente tuvo dos hospitalizaciones, ambas por falla cardíaca, y después de suspender la medicación antitiroidea. En ambas fue controlada con digitalización y medicación antitiroidea. En la última crisis cursaba 32 semanas de gestación, y después de compensarse, se llevó a 34 semanas. Se detectó un oligoamnios y sufrimiento fetal por lo cual se le realizó una operación cesárea, obteniéndose un RN femenino, apgar de 8-9 y un peso de 2.300 grs, considerado adecuado para la edad gestacional.

Seis de 30 pacientes no necesitaron medicación y cursaron asintomáticas. Cuatro de ellas con diagnóstico de E. de Graves y dos con tiroiditis. Entre estas pacientes, dos tenían diagnóstico previo al embarazo y a cuatro se les hizo durante su gestación. Todas tenían aumẹnto del tamaño de la tiroides. Las diagnosticadas como Graves además presentaban exoftalmos de diferente magnitud. Todas tenían menos de 100 de pulso (78-98). El rango de niveles de T4 y T3 fueron de 15 a $24 \mathrm{mcg} / \mathrm{dl}$ y $220-270 \mathrm{ng} / \mathrm{dl}$ respectivamente. Todos los partos llegaron al término, el apgar al nacer fue normal y el peso fue superior a 2.500 grs (rango, 2.900-3.500). Cinco terminaron su embarazo por parto vaginal. La paciente restante, con diagnóstico de tiroiditis y embarazo prolongado, fue llevada a cesárea. Todos los recién nacidos tuvieron examen físico y neurológico normal. Una paciente se complicó con toxemia y dos $(40 \%)$ con enfermedad mental (Tabla 1).

Tabla 1

HIPERTIROIDISMO Y EMBARAZO. PACIENTES SIN TRATAMIENTO

Diagnóstico, valores hormonales, relación edad del embarazo y examen del RN, peso, relación con la edad gestacional, apgar y vía de terminación del embarazo

\begin{tabular}{|lccccccccc|}
\hline DX & T4 & T3 & CP & EG/ERN & PESO & P/EG & APGAR & VT & TTO \\
G & 15 & 208 & 5 & $40 / 40$ & 2960 & A & $8-10$ & Pe & - \\
G & 24 & 270 & 3 & $40 / 40$ & 3000 & A & $8-10$ & Pe & - \\
G & 17 & 245 & 6 & $40 / 40$ & 3240 & A & $8-9$ & Pe & - \\
G & 16 & 257 & 2 & $40 / 40$ & 2900 & A & $8-8$ & Pe & - \\
T & 15 & 230 & 7 & $40 / 40$ & 3500 & A & $8-9$ & C & - \\
T & 15 & 213 & 6 & $40 / 40$ & 3280 & A & $8-8$ & Pe & - \\
\hline
\end{tabular}

G, Graves; T, Tiroiditis; CP, Consulta Prenatal; EG/ERN, Edad Gestacional/Edad RN: VT, Vía Terminación; A, Adecuado para la edad gestacional.
En las pacientes sintomáticas, los dos síntomas más frecuentes al momento del diagnóstico fueron taquicardia, frecuencia del pulso por encima de 100 por minuto e insomnio.

En la Enfermedad de Graves, los signos predominantes fueron aumento del tamaño de la glándula y exoftalmos.

Exoftalmos se encontró en el $85 \%$ de las pacientes con Graves. El $70 \%$ presentaban bocio difuso.

Las 3 pacientes diagnosticadas como hipertiroidismo, presentaban bocio difuso y taquicardia en el $100 \%$ de los casos. Ninguna tenía exoftalmos. En la historia clínica no se les catalogó como E. de Graves, por lo tanto las colocamos en un grupo aparte.

En las pacientes con tiroiditis, $60 \%$ presentaban bocio difuso y $20 \%$ bocio nodular (Figura 3 ).

Figura 3

HIPERTIROIDISMO - SINTOMAS

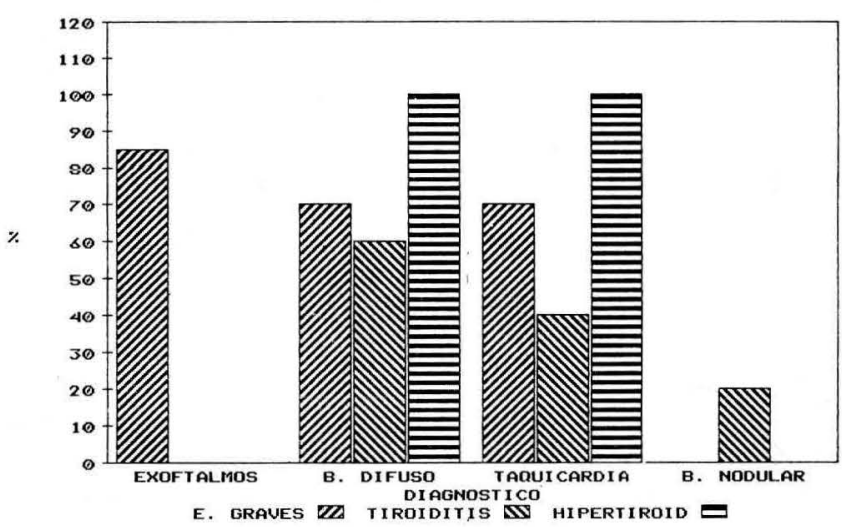

De las 30 pacientes hipertiroideas, el $65 \%$ son remitidas con el diagnóstico. En el $35 \%$ se hace durante la gestación.

En las pacientes que requieren tratamiento, $6.6 \%$ empeoran, $26.1 \%$ mejoran y $67.2 \%$ transcurren estables, cuando el parámetro es la evolución de la sintomatología.

No hubo correlación demostrable entre el número de controles prenatales, evolución del embarazo, terminación del mismo y características del recién nacido.

Sólo $2(14 \%)$ recién nacidos pesaron por debajo del esperado para la edad gestacional en el grupo de $\mathrm{E}$. de Graves.

Es notorio que los RN de las pacientes diagnosticadas como tiroiditis, el $70 \%$ pesan más de 3.000 grs, y de éstos la mitad pesa más de 3.500 grs (Figura 4).

Se realizaron 7 (23\%) cesáreas. Tres por causas obstétricas y 4 por sufrimiento fetal, dos de ellas con embarazo prolongado y otro prematuro con retardo en el crecimiento y oligoamnios marcado (Tabla 2 ).

La única mortalidad perinatal se refiere a la madre hipertiroidea, que muere cursando un embarazo de 24 semanas, con feto in útero. Esto resulta en una mortalidad perinatal del 3.3 y 1.9 por ciento en hipertiroidismo en particular y enfermedad tiroidea en general, respectivamente.

De las 24 pacientes que recibieron tratamiento, 10 reciben sólo metimazol; 11 reciben metimazol más propanolol y 3 sólo propanolol. Aunque no hay una relación matemática entre los niveles de hormona tiroidea y las dosis de medica- 
Figura 4

HIPERTIROIDISMO - PESO RN X DX

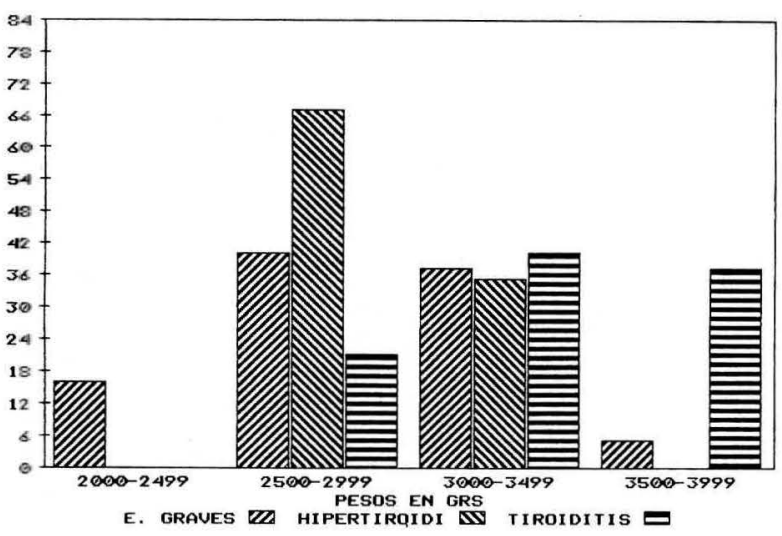

Tabla 2

HIPERTIROIDISMO

Resumen de los pacientes llevados a operación cesárea

\begin{tabular}{|cccccccccc|}
\hline D & T4 & T3 & CP & EG/ERN & Peso RN & PN & APGAR & T/P & CAUSA \\
\hline G & 24 & 230 & 3 & $36 / 36$ & 2300 & A & $8-9$ & $30 / 120$ & Oligoam SF \\
G & 29 & 315 & 2 & $40 / 40$ & 2400 & B & $8-8$ & $60 / 160$ & Transver \\
G & 20 & 280 & 1 & $40 / 40$ & 3400 & A & $8-10$ & $15 / 30$ & DisPel \\
G & 32 & 331 & 5 & $40 / 40$ & 2790 & A & $9-10$ & $30 / 40$ & SF \\
G & 14 & 367 & 4 & $40 / 40$ & 3500 & A & $10-10$ & $0 / 80$ & PrCara \\
T & 15 & 230 & 7 & $40 / 40$ & 3500 & A & $8-9$ & TTO- & E. Prol SF \\
T & 24 & 286 & 4 & $40 / 40$ & 3500 & A & $8-10$ & $5 / 0$ & E. Prol SF \\
\hline
\end{tabular}

G, Graves; T, Tiroiditis; CP, Control Prenatal; EG/ERN, Edad Gestacional/Examen RN; A, Adecuado; B, Bajo; T/P, Tapazol/Propanolol; SF, Sufrimiento Fetal.

ción antitiroidea empleada, se puede percibir al analizar la Tabla 3, que se utiliza una dosis mayor de metimazol y/o propanolol, a mayor nivel de hormona tiroidea.

Sin embargo no observamos relación directa y constante entre niveles de hormona y dosis usada. Tampoco entre los parámetros anteriores y el desarrollo del embarazo ni del producto.

Tabla 3

NIVELES DE T4 (MCG/DL) Y DOSIS DE METIMAZOL/PROPANOLOL.

Relación entre niveles hormonales y tratamiento-dosis en 29 mujeres hipertiroideas embarazadas. TTO-, sin tratamiento

\begin{tabular}{|c|c|c|c|c|c|}
\hline \multicolumn{2}{|c|}{$\begin{array}{c}14-19 \\
\mathrm{mcg} / \mathrm{ml}\end{array}$} & \multicolumn{2}{|c|}{$\begin{array}{c}20-29 \\
\mathrm{mcg} / \mathrm{ml}\end{array}$} & \multicolumn{2}{|c|}{$\begin{array}{c}30-39 \\
\mathrm{mcg} / \mathrm{ml}\end{array}$} \\
\hline T4 & $\mathbf{M} / \mathbf{P}$ & T4 & M/P & T4 & M/P \\
\hline 18 & $15 / 0$ & 24 & $30 / 120$ & 32 & $30 / 0$ \\
\hline 16 & $30 / 0$ & 25 & $0 / 40$ & 34 & $15 / 30$ \\
\hline 14 & $0 / 80$ & 20 & $30 / 0$ & 37 & $15 / 30$ \\
\hline 15 & TTO- & 29 & $60 / 160$ & 32 & $30 / 40$ \\
\hline 17 & TTO- & 20 & $0 / 35$ & 32 & $40 / 0$ \\
\hline 16 & TTO- & 22 & $30 / 80$ & 41 & $40 / 40$ \\
\hline 12 & $20 / 0$ & 24 & TTO- & & \\
\hline 14.5 & TTO- & 23 & $25 / 40$ & & \\
\hline 14.7 & TTO- & 22 & $25 / 0$ & & \\
\hline 18 & $15 / 80$ & 21 & $15 / 80$ & & \\
\hline 16.6 & $30 / 0$ & 24 & $5 / 0$ & & \\
\hline 9.8 & $30 / 0$ & & & & \\
\hline
\end{tabular}

En las pacientes que reciben metimazol propanolol, la dosis del primero es en promedio reducido a 20 mgrs o menos con relación a las que reciben sólo metimazol.

\section{Bocio nodular eutiroideo}

A cinco pacientes se les diagnosticó bocio nodular eutiroideo durante el embarazo. A una de las pacientes, previo al embarazo, se le diagnosticó un bocio tóxico nodular, el cual se trató con tiroidectomía subtotal. Sus pruebas tiroideas fueron antes a embarazarse de $19 \mathrm{mcg} / \mathrm{dl}$ y $551 \mathrm{ng} / \mathrm{dl} \mathrm{de}$ T4 y $\mathrm{T} 3$ respectivamente; al inicio del embarazo llegaron a valores de $12.1 \mathrm{mcg} / \mathrm{dl}$ y $221 \mathrm{ng} / \mathrm{dl}$. Ninguna recibió tratamiento durante el embarazo. No hubo evidencia de crecimiento de la glándula durante la gestación, aunque no se hizo un seguimiento detallando medidas exactas. Las pacientes cursaron asintomáticas con pulso normal, siempre menor de 90 y mayor de 70 latidos por minuto. Todos los embarazos alcanzaron las 40 semanas y el peso de los recién nacidos fue mayor de 3.500 grs (Tabla 4).

Tabla 4

CARACTERISTICAS DE 5 PACIENTES EMBARAZADAS CON BOCIO EUTIROIDEO

\begin{tabular}{|lcccccl|}
\hline \multicolumn{1}{|c}{ Tipo } & T4 & T3 & TSH & EG & Peso & Observaciones \\
\hline Nodular & 11 & 230 & 1.1 & $40 / 40$ & 3580 & Tiroidectomía Sub. \\
Nodular & 11 & 193 & ND & $40 / 40$ & 3860 & Preclampsia \\
Difuso & 12 & 182 & 0.4 & $40 / 40$ & 4100 & HTA \\
Nodular & 12 & 246 & 0.2 & $40 / 40$ & 3600 & Tiroidectomía Sub. \\
Nodular & & & & & & \\
Parenquimatoso & 10 & 146 & ND & $40 / 40$ & 3550 & \\
\hline
\end{tabular}

\section{Hipotiroidismo}

De las 52 pacientes con enfermedad tiroidea y embarazo, 17 fueron hipotiroideas. Se diagnosticaron así: 7 hipotiroidismos primarios; 5 tiroiditis de Hashimoto; 3 hipotiroideas secundarias a tiroidectomía: 2 por carcinoma papilar de tiroides y 1 debido a bocio hiperfuncionante; 1 hipotiroidismo post-administración de yodo radiactivo utilizado como tratamiento de hipertiroidismo por Enfermedad de Graves; 1 hipotiroidismo congénito con retardo mental.

El diagnóstico hormonal se hizo por determinación de TSH que se encontró en un rango de 8-150 mUI (Figura 5).

La principal sintomatología en pacientes sin medicación tiroidea fue intolerancia al frío, pereza, sueño y constipación (Cuadro 1).

Dos de las 17 pacientes tuvieron su parto en diferentes ciudades del país y no se conoce el resultado. Las 15 restantes llevaron su embarazo a término y el peso del recién nacido fluctuó entre 2.700 y 3.900 grs. Todos los recién nacidos fueron catalogados con peso adecuado para la edad gestacional.

Dos pacientes con diagnóstico de hipotiroidismo y antecedentes de tratamiento con hormona tiroidea, no necesitaron tratamiento durante el embarazo actual, y transcurrieron asintomáticas.

No hubo morbilidad ni mortalidad fetal. Tampoco hubo mortalidad materna.

Cuatro (23\%) pacientes terminaron en cesárea, todas por causa obstétrica: 3 distocias pélvicas y una por dos cesáreas previas. 
La mitad de las pacientes tratadas recibieron Eutrode y la otra mitad Cynomel, $200 \mathrm{mcg}$ y $50 \mathrm{mcg}$ respectivamente.

Todas las pacientes fueron diagnosticadas como hipotiroideas antes del embarazo.

Figura 5

HIPOTIROIDISMO - PERFIL HORMONAL

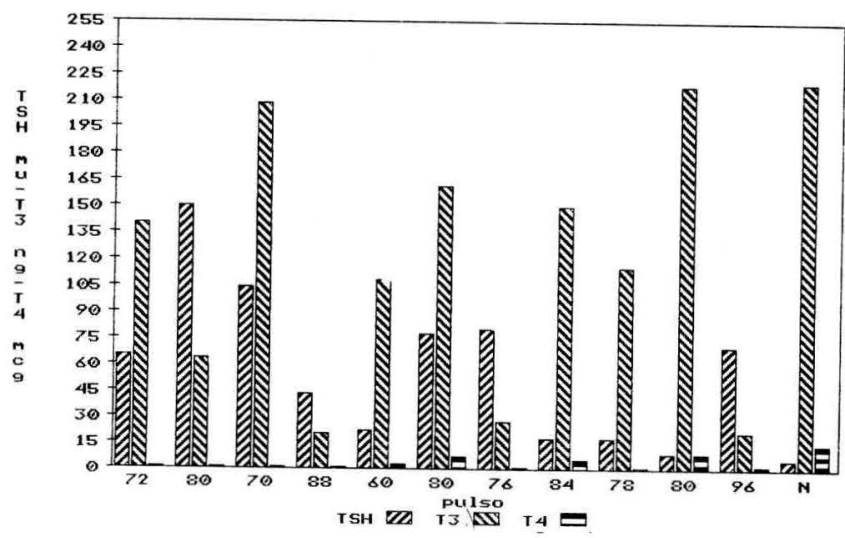

Niveles de TSH - T3 - T4 en pacientes hipotiroideas embarazadas al momento del diagnóstico. Relación con frecuencia del pulso por minuto.

Cuadro 1

PREVALENCIA DE SIGNOS Y SINTOMAS EN HIPOTIROIDISMO EN 17 PACIENTES EMBARAZADAS

$\%$

\begin{tabular}{|lc|}
\hline Intolerancia al trio & 35.0 \\
Pereza & 41.1 \\
Constipación & 29.4 \\
Sueño & 35.2 \\
Adinamia & 17.6 \\
Anemia & 17.6 \\
\hline
\end{tabular}

\section{Discusión}

El estado hiperdinámico del embarazo, manifestado por taquicardia, aumento del gasto cardíaco que lleva a amplitud del pulso y soplo sistólico de eyección; eritema palmar, intolerancia al calor y fatiga, puede confundir el diagnóstico clínico de hipertiroidismo.

El aumento de la depuración de yodo, debido a un incremento de la filtración glomerular, conduce a un aumento del tamaño de la glándula tiroidea, considerado como normal y fisiológico en el embarazo.

Los requerimientos de yodo en la dieta son de $250 \mathrm{mcgs} /$ día. La deficiencia de ingesta de yodo incrementa los niveles de TSH, que estimula la captación de yodo con aumento secundario del tamaño de la glándula.

La mujer es normalmente eutiroidea durante el embarazo, a pesar de un incremento en el metabolismo basal (26-27). El mayor efecto del embarazo sobre las pruebas tiroideas es el resultado del incremento de la TBG, que a su vez es resultado del incremento de estrógenos, con efecto final de aumento de los niveles de T3 y T4 unida a las proteínas transportadoras.

En cuanto a TSH, en el embarazo temprano está disminuida (posiblemente secundaria al aumento de HCG), pero posteriormente se normaliza (28-29).
La Enfermedad de Graves es considerada como un trastorno autoinmune primario, caracterizado por la producción de anticuerpos estimulantes del tiroides (TSAb), dirigidos contra los receptores de TSH en la célula tiroidea. Los TSAb compiten con la TSH y estimulan la producción excesiva de T4 y T3, con un cuadro de hipertiroidismo como resultado. Se caracteriza también por remisiones y exacerbaciones durante la vida, con una mayor incidencia en la edad reproductiva.

La patogénesis completa de esta enfermedad no está del todo aclarada, y la causa de la enfermedad se desconoce. Se invocan causales hereditarios, emocionales, infecciosos y del sistema nervioso simpático, como importantes factores etiológicos. Adams y Purves describieron, en 1956, un estimulador tiroideo de acción prolongada (LATS), en el suero de pacientes con Graves. Posteriormente en animales se demostró la acción tiroide estimulante del suero de pacientes con esta enfermedad. Más tarde se probó que LATS era un anticuerpo contra un antígeno tiroideo, presente en una inmunoglobulina del tipo $\operatorname{IgG}(10)$.

En los años 70 se desarrollaron diversos ensayos para detectar la presencia de anticuerpos estimulantes tiroideos. Los ensayos están basados en la estimulación del sistema de AMP cíclico en pequeños cortes de tejido o membranas tiroideas. Para su denominación se ha usado una diversa y caótica terminología entre la cual se encuentra: HTS, estimulante tiroideo humano; H-TACS, estimulante de la adelnilciclasa tiroidea humana; TSAb, anticuerpos estimulantes del tiroides; TSI, inmunoglobulina estimulante del tiroides; y estimulante tiroideo de larga acción LATS, entre otros.

El trabajo de muchos laboratorios indica que TSAb es el anticuerpo estimulante tiroideo relacionado con el hipertiroidismo de la Enfermedad de Graves. Sin embargo no se ha podido definir si este es el factor causal o sólo un marcador inmunológico de la enfermedad.

En los embarazos complicados con Enfermedad de Graves, TSAb cruza la barrera placentaria y estimula la tiroides fetal. Si el título de TSAb es lo suficientemente alto, puede desarrollarse un hipertiroidismo fetal en un lapso de 24 meses (11).

Aún no se dispone comercialmente de los test para medir antiglobulinas tiroideas. Sin embargo se han hecho varias observaciones clínicas durante el embarazo: a) títulos altos de TSAb están más relacionados con hipertiroidismo neonatal, que cuando estos títulos son bajos; b) los títulos de LATS pueden ser negativos con títulos positivos de TSAb; c) el recién nacido con E. de Graves puede tener hipertiroidismo persistente por años después de pasar la vida media biológica de los anticuerpos antitiroideos; d) se han descrito varios recién nacidos con Graves en los cuales no hay historia de enfermedad tiroidea en la madre; e) también hay informes de niños con Graves neonatal cuyas madres presentan títulos negativos para LATS y LATS-P, pero positivos para TSAb (12-13).

La mayoría de nuestras pacientes ingresan a la clínica con diagnóstico de hipertiroidismo previo al embarazo. El diagnóstico durante la gestación, especialmente en los estados tempranos de la enfermedad, no es fácil de hacer. La embarazada normal puede presentar irritabilidad, labilidad emocional, intolerancia al calor y aumento de la perspira- 
ción. En nuestra experiencia los síntomas predominantes son taquicardia, palpitaciones e insomnio. Estos síntomas asociados a aumento del tamaño de la glándula, de manera difusa o nodular con o sin exoftalmos, justifican la determinación de T4 y T3. Valores iguales o superiores a $15 \mathrm{mcg} / \mathrm{dl}$ y $220 \mathrm{ng} / \mathrm{dl}$ respectivamente, hacen el diagnóstico.

La determinación de T4 y T3 libre confirman la hiperproducción de hormonas tiroideas. Indirectamente, la determinación de captación de T3 por resina (T3 upT) en niveles normales altos, nos informa de la excesiva ocupación de los receptores de la tiroglobulina por un exceso de hormona tiroidea en su forma libre. Donde hay disponibilidad de la medición de TSAb, pronosticaría los fetos a riesgo de desarrollar hipertiroidismo in útero.

El diagnóstico etiológico en nuestro medio fueron la Enfermedad de Graves y la fase hipermetabólica de la tiroiditis de Hashimoto. La primera fue hecha inequívocamente cuando la paciente presentaba la tríada de bocio difuso, síndrome ocular y tirotoxicosis. Las pacientes que no presentaron exoftalmos, aunque con bocio difuso y elevación de las hormonas tiroideas, se catalogaron como hipertiroideas, pero podrían corresponder a una tiroiditis aguda (subaguda) con hipertiroidismo transitorio. Esta última está reportada en la literatura como la de mayor frecuencia en el embarazo (14). Todas las pacientes con enfermedad de Hashimoto fueron diagnosticadas por biopsia, lo cual es importante porque a veces es difícil de diferenciar entre la fase hipermetabólica de la enfermedad de Hashimoto y la E. de Graves, coexistiendo en algunos casos.

Se ha descrito una alta incidencia de mortalidad perinatal y una significante mortalidad neonatal en madres hipertiroideas no tratadas. También se ha descrito bajo peso para la edad gestacional (30-31). Nuestra mortalidad perinatal es de $3.3 \%$ y se reduce a la muerte materna concomitantemente con feto in útero. En ambos casos es lo esperado: que se reduzca la mortalidad perinatal y neonatal, cuaiddo se controla la enfermedad, y por el contrario una mortalidad materna y fetal muy alta en la crisis hipertiroidea. Es evidente el bajo peso del RN en los pacientes con hipertiroidismo. El peso de los RN en madres con tiroiditis de Hashimoto y el de éstos en relación con las pacientes con bocio eutiroideo, comparados con las hipertiroideas con E. de Graves, es indiscutiblemente superior.

Cuatro pacientes fueron llevadas a operación cesárea por sufrimiento fetal. Es decir, en el $57 \%$ de las cesáreas hay compromiso del feto y son realizadas por esa causa. En una de ellas a la semana 36 de gestación hay signos de sufrimiento fetal (Tabla 2). Lo anterior justifica el inicio de monitoría fetal a partir de la semana 32 ó 34 de gestación y control ecográfico para determinar bienestar fetal, peso fetal y cantidad de líquido amniótico. También implica como norma que estas pacientes deben tener partos monitorizados.
El objetivo de la terapia es alcanzar un estado normal o ligeramente hipertiroideo en la madre. Como es obvio, también es importante el resultado perinatal. Debido a que el propiltiuracilo atraviesa más lentamente la barrera placentaria que el metimazol, y además inhibe la conversión periférica de T4 a T3, se considera la droga de elección.

En todos nuestros pacientes utilizamos metimazol como medicación de primera línea. El propanolol lo utilizamos para disminuir la dosis de metimazol y en las pacientes con síntomas cardiovasculares muy manifiestos. Nuestra experiencia con metimazol, con resultados perinatales buenos, la hacen una medicación segura. No utilizamos propanolol como única medicación durante la gestación. Se ha descrito con su uso retardo del crecimiento (21-25), bradicardia (22), policitemia (23), hipocalcemia (21), hiperbilirrubinemia (21) y apnea neonatal (21-24). El hecho de no haber encontrado morbilidad en nuestros recién nacidos, atribuible al propanolol, lo explicamos así: instauramos la medicación cuando no es posible controlar el paciente con hasta $40 \mathrm{mgs}$ de metimazol, la dosis es menor y el tiempo de exposición también.

Como algunos autores lo han reportado, no hemos encontrado hipersensibilidad o reacciones idiosincrásicas con el uso de metimazol (14).

El diagnóstico de hipotiroidismo, en la gran mayoría de los casos, es hecho previo al embarazo. El embarazo espontáneo, en una mujer hipotiroidea, es extremadamente raro. Desde 1897 han sido reportados 36 casos de hipotiroideas que se embarazan y en la mayoría de esas pacientes el diagnóstico ha sido hecho por sospecha clínica (15-16). La paciente usualmente consulta a la clínica de infertilidad por incapacidad para lograr un embarazo o por abortos a repetición (17). Más o menos el 5\% de las pacientes hiperprolactinémicas tienen hipotiroidismo clínico o subclínico. El resto de las pacientes son clasificadas como hipotiroideas primarias o tiroiditis de Hashimoto, en las que tienen biopsia de la glándula. Los hipotiroidismos secundarios se presentan como secuela del tratamiento del Carcinoma tiroideo.

El manejo de la paciente hipotiroidea, una vez embarazada, es relativamente fácil y termina sin mayores complicaciones materno-fetales (6). El remplazo con hormona tiroidea, monitorizado con TSH, que llega a niveles normales ( $5 \mathrm{mUL}$ ), la desaparición de la sintomatología subjetiva y la normalización del pulso (70-80 por min.) son los parámetros básicos para el control. El manejo obstétrico no requiere individualización. Una ecografía a la semana 20 y otra en la semana 38 de gestación nos dan la información requerida: crecimiento intrauterino, localización y madurez placentaria, tamaño y posición fetal, malformaciones detectables y características de líquido amniótico. Por último, la vía de terminación del embarazo depende de la evaluación obstétrica que se realice.

\section{BIBLIOGRAFIA}

1. OPPENHEIMER, J.H. Role of plasma protein in the binding distribution and metabolism of the thyroid hormones. NEngl J Med 1968;278: 1153.

2. BURGUS, R.; GUILLEMAN, R. Hypotalamic releasing factors. Ann Rev Biochem 1970; 39: 499.

3. SCHALLY, A.V.; REDDING, T.W.; BOWERS, C.Y.; BARRETT,
J.F. Isolation and properties of porcine thyrotropin releasing hormone. J Biol Chem 1969; 244: 4077

4. BURROW, G.N. Hyperthyroidism during pregnancy. N Engl J Med 1978; 298: 150.

5. ORMSTON, B.J.; GARRY, R.; CRYER, R.J.; BESSER, G.M., 
HALL, R. Thyrotropin releasing hormone as a thyroid function test. Lancet 1970; 2: 10.

6. MONTORO, M.; COLLEÀ, J.V.; FRASIER, S.D.; MESTMAN, J.H. Succesfull outcome of pregnancy in women with hypothyroidism. Ann Intern Med 1981; 94: 31 .

7. BURROW, G.N. The thyroid gland and reproduction. In Yen SSC, Jaffe RB, eds. Reproductive Endocrinology. Philadelphia: W.B. Saunders Co. 1978; 373.

8. PARKIN, G.; GREENE, J.A. Pregnancy occurring in cretinism and in juvenile and adult myxedema. J Clin Endocrinol Metab 1943; 3: 446.

9. ANDERSON, M.M.; BEALES, D.L. Myxedema in pregnancy. J Obstet Gynecol Br Commonw 1970; 77: 74.

10. McKENZIE, J.M.; ZAKARIJA, M.; SATO, A. Humoral, inmunity in Graves' disease. Clin Endocrinol Metab 1978; 7: 31.

11. FURTH, E.D. Thyroid and Parathyroid hormone function in pregnancy in Endocrinology of Pregnancy ed. Fuchs F, Klopper A. pág. 176.

12. MUNRO, D.S.; DIRMIKIS, S.M.; HUMPHRIES, H.; SMITH, T.; BROADHEAD, G.D. The role of thyroid stimulating inmunoglobulins of Graves' disease in neonatal thyrotoxicosis. Br J Obstet Gynecol 1978; 85: 837.

13. WILROY, R.S.; ETTELDORF, J.N. Familial hyperthyroidism including two siblings with neonatal Graves' disease. J Pediatr 1971; 625.

14. HOLLINGSWORTH, D.R. Graves' Disease. Clin Obstet Gynecol 1983; Vol. 26 No 3, 615.

15. ANDERSON, M.M.; BEALES, D.L. Myxedema in pregnancy. J Obstet Gynecol Br Commonw 1970; 77: 74.

16. LACHELIN, G.C.L. Myxedema and pregnancy, a case report. J Obstet Gynecol Br Commonw 1970; 77: 77.

17. PRITCHARD, J.A.; MacDONALD, P.C. Williams Obstetrics. 15th ed. New York: Appleton-Century Crofts; 1976; 627.

18. CHERON, R.G.; KAPLAN, M.M.; LARSEN, P.R.; SELENKOW,
H.A.; CRIGLER, J.F. Neonatal thyroid function after propylthiouracil therapy for maternal Graves' disease. N Engl J Med 1981; 304: 525.

19. BURROW, G.N. Neonatal goiter after maternal propylthiouracil therapy. J Clin Endocrinol Metab 1965; 25: 4039.

20. BURROW, G.N.; BARSOCAS, C.; KLATSKIN, E.H.; GRUNT, J.A. Children exposed in utero to propylthiouracil. Am J Dis Child 1968; 161.

21. FIDDLER, G.I. Propanolol and pregnancy. Lancet 1974; 2: 722.

22. DATTA, S.; KITZMILLER, J.L.; OSTHEIMER, G.W.; SHOEMBAUM, S.C. Propanolol and parturation. Obstet Gynecol 1978; $51: 577$.

23. HABIB, A.; McCARTHY, J.S. Effects, on the neonate of propanolol administered during pregnancy. J Pediatr 1977; 91: 808.

24. TURNSTALL, M.B. The effect of propanolol on the onset of breathing at birth. Br J Anesth 1969; 51: 792.

25. PRUYN, S.C.; PHELAN, J.P.; BUCHANAN, G.C. Long term propanolol therapy in pregnancy: Maternal and Fetal outcome. Am J Obstet Gynecol 1979; 135: 485.

26. ABOUL-KHAIR, S.A. et al. The physiological changes in thyroid function during pregnancy. Clin Sci 1964; 27: 195.

27. LEVY, R.P.; NEWMAN, D.M.; LEJALI, L.S.; BARFORD, D.A.G. The myth of goiter in pregnancy. Am J Obstet Gynecol 1980; 137: 701.

28. YAMAMOTO, $\mathrm{T}$. et al. Longitudinal study of serum thyroid hormones. chorionic gonadotrophin and thyrotrophin during and after normal pregnancy. Clin Endocrinol 1979: 10: 459

29. HARADA, A. et al. Comparison of thyroid stimulators and thyroid hormone concentrations in the sera of pregnant women. J Clin Endocrinol Metab 1979; 48: 793

30. MESTMAN, J.H.; MANNING, P.R.; HODGMAN, J. Hyperthyroidism and pregnancy. Arch Int Med 1974: 134: 434

31. MONTORO, M.; MESTMAN, J.H. Graves disease and pregnancy. NEJM 1981; 305: 48.

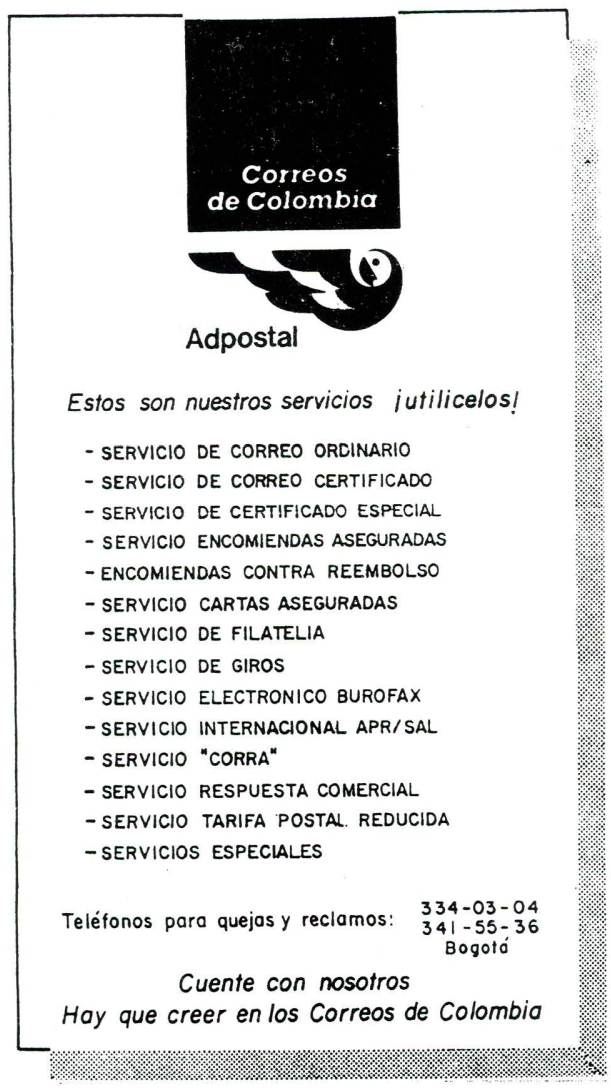

